\title{
Descripción de dos renacuajos y una clave para las larvas conocidas del grupo Bufo spinulosus (Anura: Bufonidae) de Perú
}

\author{
Description of two tadpoles and a key to the known larvae of the Bufo \\ spinulosus group (Anura: Bufonidae) occurring in Peru
}

\author{
César Aguilar* y Rocío Gamarra
}

Presentado: $27 / 05 / 2004$

Aceptado: $\quad 03 / 08 / 2004$

\section{Resumen}

Se describen los renacuajos de Bufo arequipensis y Bufo cophotis. Ningún carácter externo examinado permite distinguir la larva de las especies nominales $B$. arequipensis, $B$. flavolineatus y $B$. trifolium. La larva de Bufo limensis se diferencia de las de $B$. flavolineatus y $B$. trifolium por presentar un espacio menor en la segunda fila anterior de dentículos labiales y una fórmula de dentículos labiales (LTRF) igual a 2(2)/3[1]. B. cophotis difiere de B. limensis, B. flavolineatus y B. trifolium por presentar la altura máxima de la cola en su extremo posterior, una coloración marrón oscura en el cuerpo y cola, y un LTRF igual a 2(2)/3(1). Se proporciona una clave de identificación para las larvas conocidas del grupo spinulosus presentes en Perú.

Palabras clave: Renacuajos, Bufo arequipensis, Bufo cophotis, grupo Bufo spinulosus, Perú.

\section{Abstract}

The tadpoles of Bufo arequipensis and Bufo cophotis are described. No external larval character studied allowed the distinction between the tadpoles of the nominal species $B$. arequipensis, $B$. flavolineatus and $B$. trifolium. The tadpole of $B$. limensis differs from the ones of $B$. flavolineatus and $B$. trifolium by the presence of a small gap in the second anterior tooth row and a 2(2)/3[1] labial tooth row formula (LTRF). The tadpole of $B$. cophotis differs from B. limensis, $B$. flavolineatus and $B$. trifolium larvae by showing the highest part of the tail in its posterior end, a dark brown coloration in the body and tail, and a 2(2)/3(1) LTRF. An identification key for the known Peruvian tadpoles of the spinulosus group is provided.

Keywords: Tadpoles, Bufo arequipensis, Bufo cophotis, Bufo spinulosus group, Peru.

\section{Introducción}

El género Bufo Laurenti se considera compuesta de 252 especies, de las cuales más de 50 están presentes en Sudamérica (Frost, 2002). Las especies de Bufo presentes en Sudamérica han sido clasificadas fenéticamente en seis grupos (Duellman y Schulte, 1992). De éstos los más diversos en Perú son los grupos $B$. spinulosus y $B$. veraguensis (Duellman y Schulte, 1992; Frost, 2002; Lehr, 2002); pero es el grupo Andino B. spinulosus el que ha sido más estudiado sistemáticamente tanto a nivel del reconocimiento de especies como en

\footnotetext{
* Museo de Historia Natural, Universidad Nacional Mayor de San Marcos, Av. Arenales 1256, Jesús María, Apartado 14-0434, Lima, Perú.

E mail César Aguilar: caguilarp@unmsm.edu.pe
}

sus relaciones filogenéticas. Con respecto al reconocimiento de especies de $B u f o$, el cátalogo de Frost (2002) lista para Perú 8 especies nominales dentro de este grupo (a diferencia de Lehr (2002) que lista 5) considerando como válidas a $B$. arequipensis Vellard, $B$. cophotis Boulenger, B. corynetes Duellman y Ochoa, B. flavolineatus Vellard, B. limensis Werner, B. spinulosus Wiegmann, B. trifolium (Tschudi) y B. vellardi Leviton y Duellman; no obstante la evidencia proveniente de diferentes complejos de caracteres como la osteológica, molecular (albúminas y alozimas), cromosómica, bioacústica, larval, de historia de vida y biología reproductiva (Martin, 1972; Maxon, 1984; Sinsch, 1986; 1990; Córdova, 1999; Haas, 2002) sugiere que las 
Tabla 1. Número de especímenes examinados por especie, localidad y estadio.

\begin{tabular}{|c|c|c|c|c|c|c|c|c|c|c|}
\hline \multirow[t]{2}{*}{ Especie nominal } & \multirow[t]{2}{*}{ Localidad } & \multicolumn{9}{|c|}{ Estadio de Gosner (1960) } \\
\hline & & 29 & 33 & 34 & 35 & 36 & 37 & 38 & 39 & 40 \\
\hline Bufo arequipensis & Yura, Arequipa & & & 2 & 4 & 3 & 1 & & & \\
\hline \multirow{2}{*}{ Bufo cophotis } & Huaychopampa, Ancash & & & & & 1 & & & & 3 \\
\hline & Ruricocha, Ancash & & & & & 2 & 4 & & & \\
\hline \multirow[t]{2}{*}{ Bufo limensis } & Barba blanca, Lima & & & & 1 & & 1 & & & \\
\hline & Río Pisco, Ica & 1 & & 1 & 1 & 3 & 1 & & & 1 \\
\hline Bufo flavolineatus & Accochaca, Lima & & & & & 2 & & 1 & & 2 \\
\hline \multirow{2}{*}{ Bufo trifolium } & Paucartambo, Pasco & & 1 & 1 & 1 & & & 4 & & \\
\hline & Huamanga, Ayacucho & & & & & 1 & & 2 & & \\
\hline
\end{tabular}

anteriormente reconocidas especies $B$. flavolineatus y B. trifolium (Cei, 1972; Duellman y Schulte, 1992) deberían ser tratados como sinónimos de $B$. spinulosus. Del mismo modo, la evidencia cromosómica también indica que $B$. arequipensis debería ser un sinónimo de B. spinulosus (Córdova, 1999). Sin embargo, los caracteres larvales no han sido utilizados en la delimitación de las especies del grupo debido a que sólo los renacuajos de $B$. spinulosus (que incluiría a $B$. flavolineatus у B. trifolium) у B. limensis han sido descritos (Cei, 1980; Sinsch, 1986; Sinsch, 1990; Haas, 2002; Angulo y Aguilar, 2003).

Por estos motivos los objetivos en el presente trabajo son: 1) describir las larvas de $B$. arequipensis y $B$. cophotis, 2) comparar la morfología externa larval de las especies nominales $B$. arequipensis, $B$. cophotis, $B$. limensis, $B$. flavolineatus y B. trifolium y discutir la importancia de la morfología larval en la discriminación de las especies peruanas del grupo spinulosus, y 3) presentar una clave para las larvas conocidas de las especies peruanas del grupo spinulosus.

\section{Material y métodos}

Las especies nominales, número de especímenes y estadios larvales examinados, así como sus localidades se muestran en las Tablas 1 y 2. Los renacuajos fueron fijados en formalina al 10\% y están depositados en el Departamento de Herpetología, Museo de Historia Natural de San Marcos (MHNSM), Lima.

Tabla 2. Información sobre las especies nominales examinadas en el presente trabajo.

Bufo arequipensis. Los renacuajos examinados pertenecen a la serie larval: MUSM 19322 (diez especímenes) colectada e identificada por César Aguilar y Elías Ponce en Yura, provincia Arequipa, departamento Arequipa, en abril de 2000 .

Bufo cophotis. Los renacuajos examinados pertenecen a las series larvales: MUSM 6621 (cuatro especímenes) colectada e identificada por Antonio Salas en Huaychopampa, provincia Huari, departamento Ancash, el 15 de abril de 1989; MUSM 6622 (seis especimenes) colectada e identificada por Antonio Salas en la laguna Ruricocha, Aczo, provincia Antonio Raimondi, departamento Ancash, el 20 de abril de 1989.

Bufo limensis. Los renacuajos examinados pertenecen a las series larvales: MUSM 17987 (dos especímenes) colectada e identificada por César Aguilar en Barba Blanca, provincia de Huarochirí, departamento Lima, el 4 de abril de 1999; MUSM 19231 (ocho especímenes) colectada e identificada por César Aguilar en el río Pisco, provincia Pisco, departamento Ica, el 22 de mayo de 2001.

Bufo flavolineatus. Los renacuajos examinados pertenecen a las series larvales: MUSM 16992 (cinco especímenes) colectada e identificada por Helena Sisniegas en Accochaca, provincia Canta, departamento Lima, el 25 de marzo de 1989.

Bufo trifolium. Los renacuajos examinados pertenecen a las series larvales: MUSM 17815 (siete especímenes) colectada por Mikael Lundberg en Paucartambo, provincia Cerro de Pasco, departamento Pasco e identificada por César Aguilar; MUSM 19323(tres especímenes) colectado e identificado por César Aguilar en el Río Huatata, provincia Huamanga, departamento Ayacucho, en agosto de 2001. 

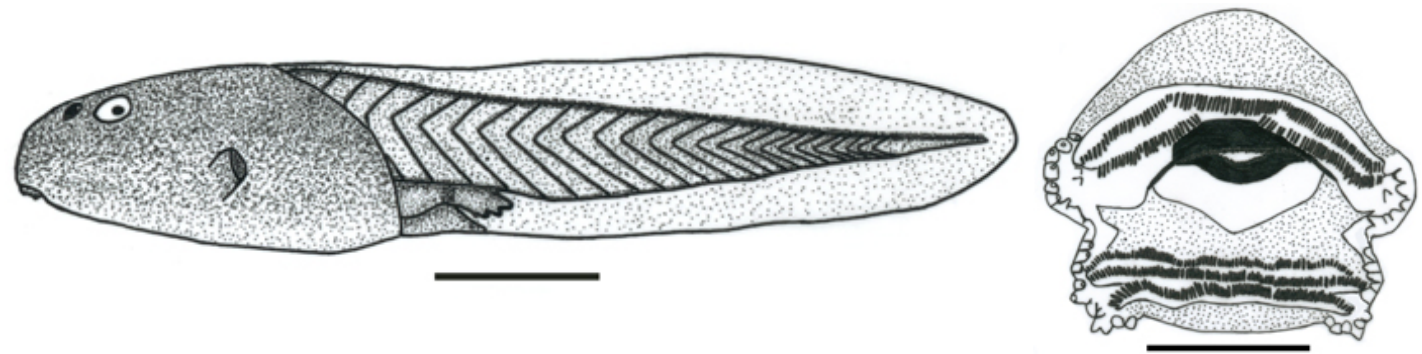

(a)

(b)

Figura 1. a) Vista lateral de la larva de Bufo arequipensis en el estadio 36 . Barra $=5 \mathrm{~mm}$. b) Vista frontal del disco oral de larva de Bufo arequipensis en el estadio 36 . Barra $=1 \mathrm{~mm}$.

Los estadios de los renacuajos fueron determinados siguiendo a Gosner (1960) (Tabla 1). Las medidas fueron tomadas con un ocular micrométrico hasta el $0,01 \mathrm{~mm}$ más cercano y los dibujos se hicieron con asistencia de un microscopio estereoscópico con cámara lúcida. La terminología de los caracteres larvales sigue a Altig y McDiarmid (1999). Debido a que se utilizaron larvas en diferentes estadios que varían en sus medidas absolutas y para hacer comparable los datos morfométricos entre especies, se usaron las siguientes proporciones (las abreviaciones entre paréntesis): longitud del cuerpo/longitud total (LC/LT), longitud de la cola/longitud total (LCO/LT), distancia ojo-narina/distancia ojo-hocico (DON/ $\mathrm{DOH})$, distancia internarial/distancia interorbital
(DIN/DIO), distancia hocico-espiráculo/longitud del cuerpo (DHE/LC), diámetro narina/ diámetro ojo (DiN/DiO), altura máxima de cola muscular/ancho máximo de cola muscular (ALMCOM/ANMCOM) y ancho del espacio en la segunda fila de dentículos del labio anterior/ancho de la segunda fila de dentículos del labio anterior (AE2F/A2F).

\section{Resultados}

\section{Descripción del renacuajo de Bufo arequipensis}

(MUSM 19322, estadio de Gosner 36)

(Fig. 1a).

Largo del cuerpo 11,3 mm, longitud total 29,3 mm. Cuerpo ovoide en vista dorsal; altura máxima del cuerpo $4,8 \mathrm{~mm}$, ancho máximo

Tabla 3. Proporciones de datos morfométricos para larvas de cinco especies nominales del grupo Bufo spinulosus. La primera fila representa el rango de las proporciones, la segunda fila muestra la media \pm la desviación estándar. Ver Material y Métodos para la abreviación de las variables.

\begin{tabular}{|c|c|c|c|c|c|c|c|c|c|}
\hline $\begin{array}{l}\text { Especie } \\
\text { nominal }\end{array}$ & $\mathbf{N}$ & LC/LT & LCO/LT & DON/DOH & $\begin{array}{c}\text { Variable } \\
\text { DIN/DIO }\end{array}$ & DHE/LC & $\mathrm{DiN} / \mathrm{DiO} \mathrm{A}$ & MCOM/ANMC & M AE2F/A2F \\
\hline B. arequipensis & 10 & $\begin{array}{c}0,37-0,45 \\
0,39 \pm 0,02\end{array}$ & $\begin{array}{c}0,55-0,62 \\
0,61 \pm 0,02\end{array}$ & $\begin{array}{l}0,29-0,38 \\
0,33 \pm 0,03\end{array}$ & $\begin{array}{c}0,56-0,70 \\
0,63 \pm 0,05\end{array}$ & $\begin{array}{c}0,58-0,60 \\
0,59 \pm 0,01\end{array}$ & $\begin{array}{c}0,25-0,44 \\
0,33 \pm 0,05\end{array}$ & $\begin{array}{c}0,68-0,80 \\
0,75 \pm 0,04\end{array}$ & $\begin{array}{c}0,17-0,29 \\
0,22 \pm 0,04\end{array}$ \\
\hline B. cophotis & 10 & $\begin{array}{c}0,41-0,46 \\
0,44 \pm 0,01\end{array}$ & $\begin{array}{l}0,54-0,59 \\
0,56 \pm 0,01\end{array}$ & $\begin{array}{c}0,25-0,38 \\
0,32 \pm 0,04\end{array}$ & $\begin{array}{l}0,48-0,58 \\
0,54 \pm 0,03\end{array}$ & $\begin{array}{l}0,42-0,57 \\
0,51 \pm 0,05\end{array}$ & $\begin{array}{c}0,33-0,75 \\
0,53 \pm 0,15\end{array}$ & $\begin{array}{c}0,63-0,82 \\
0,72 \pm 0,07\end{array}$ & $\begin{array}{l}0,18-0,29 \\
0,22 \pm 0,04\end{array}$ \\
\hline B. limensis & 10 & $\begin{array}{l}0,37-0,42 \\
0,40 \pm 0,01\end{array}$ & $\begin{array}{l}0,57-0,63 \\
0,60 \pm 0,02\end{array}$ & $\begin{array}{l}0,28-0,36 \\
0,32 \pm 0,03\end{array}$ & $\begin{array}{l}0,49-0,61 \\
0,53 \pm 0,03\end{array}$ & $\begin{array}{l}0,51-0,63 \\
0,58 \pm 0,04\end{array}$ & $\begin{array}{l}0,20-0,36 \\
0,23 \pm 0,04\end{array}$ & $\begin{array}{c}0,84-1,03 \\
0,93 \pm 0,07\end{array}$ & $\begin{array}{l}0,03-0,09 \\
0,07 \pm 0,02\end{array}$ \\
\hline B. flavolineatus & 5 & $\begin{array}{r}0,35-0,40 \\
0,37 \pm 0,02\end{array}$ & $\begin{array}{l}0,61-0,65 \\
0,63 \pm 0,02\end{array}$ & $\begin{array}{l}0,32-0,36 \\
0,35 \pm 0,02\end{array}$ & $\begin{array}{l}0,46-0,68 \\
0,55 \pm 0,08\end{array}$ & $\begin{array}{l}0,44-0,58 \\
0,54 \pm 0,06\end{array}$ & $\begin{array}{c}0,30-0,38 \\
0,33 \pm 0,04\end{array}$ & $\begin{array}{c}0,65-0,92 \\
0,75 \pm 0,11\end{array}$ & $\begin{array}{c}0,24-0,29 \\
0,26 \pm 0,02\end{array}$ \\
\hline B. trifolium & 8 & $\begin{array}{l}0,39-0,47 \\
0,41 \pm 0,03\end{array}$ & $\begin{array}{c}0,53-0,61 \\
0,59 \pm 0,03\end{array}$ & $\begin{array}{c}0,26-0,35 \\
0,32 \pm 0,03\end{array}$ & $\begin{array}{c}0,46-0,62 \\
0,51 \pm 0,05\end{array}$ & $\begin{array}{c}0,51-0,64 \\
0,59 \pm 0,04\end{array}$ & $\begin{array}{c}0,20-0,50 \\
0,32 \pm 0,10\end{array}$ & $\begin{array}{c}0,54-0,82 \\
0,71 \pm 0,08\end{array}$ & $\begin{array}{l}0,20-0,27 \\
0,22 \pm 0,03\end{array}$ \\
\hline
\end{tabular}


del cuerpo $6,0 \mathrm{~mm}$. Hocico redondeado en vista dorsal y lateral. Ojos de posición dorsal, orientados lateralmente, diámetro $0,9 \mathrm{~mm}$, distancia interorbital 2,4 mm. Narinas externas de posición dorsal, ubicadas a un tercio de la distancia de los ojos al hocico. Espiráculo sinistral y lateral, abertura del espiráculo orientado posterodorsalmente, pared interna ausente. Tubo ventral medial. Altura máxima de la cola $4,4 \mathrm{~mm}$ y ubicada aproximadamente en la mitad de su longitud, altura máxima de la cola muscular 2,5 $\mathrm{mm}$, ancho máximo de cola muscular $1,8 \mathrm{~mm}$.

Disco oral de posición ventral, emarginado (Fig. 1b). Papilas marginales presentes sólo lateralmente en el labio anterior y posterior. Una papila submarginal presente en el labio anterior. Envoltura mandibular dorsal más larga que la ventral, ambas con bordes ligeramente aserrados. Fórmula de filas labiales dentadas 2(2)/3. Las proporciones se muestran en la Tabla 3.

La coloración en líquido preservante es de manchas grisáceas pequeñas que cubren el dorso del cuerpo, menos densas lateralmente y el vientre es casi translúcido, intestino ligeramente visible; la musculatura caudal es blanco cremosa con manchas grisáceas pequeñas, las aletas son ligeramente translúcidas.

Hábitat: el lote de renacuajos fue observado en un arroyo temporal de corriente lenta y poco profundo. El sustrato del arroyo estaba formado por arena y algas verdes.

\section{Descripción del renacuajo de Bufo cophotis}

(MUSM 6621, estadio de Gosner 36)

(Fig. 2a) .

Largo del cuerpo $10,9 \mathrm{~mm}$, longitud total 23,8 mm. Cuerpo ovoide en vista dorsal; altura máxima del cuerpo $5,4 \mathrm{~mm}$, ancho máximo del cuerpo 6,1 mm. Hocico redondeado en vista dorsal y lateral. Ojos de posición dorsal, orientados lateralmente, diámetro $0,4 \mathrm{~mm}$, distancia interorbital 2,3 mm. Narinas externas de posición dorsal, ubicadas a un tercio de la distancia de los ojos al hocico. Espiráculo sinistral y lateral, abertura del espiráculo orientado posterodorsalmente, pared interna ausente. Tubo ventral medial. Altura máxima de la cola 3,9 mm y ubicada en el último tercio de la cola, altura máxima de la cola muscular $2,1 \mathrm{~mm}$, ancho máximo de cola muscular 1,6 mm.

Disco oral de posición ventral, emarginado (Fig. 2b). Papilas marginales presentes sólo lateralmente en el labio anterior y posterior. Al menos un par de papilas submarginales presentes lateralmente en el labio anterior. Envoltura mandibular dorsal más larga que la ventral, ambas con bordes ligeramente aserrados. Formula de filas labiales dentadas 2(2)/3(1). Las proporciones se muestran en la Tabla 3.

La coloración en líquido preservante es marón oscuro tanto en el cuerpo como en la cola.

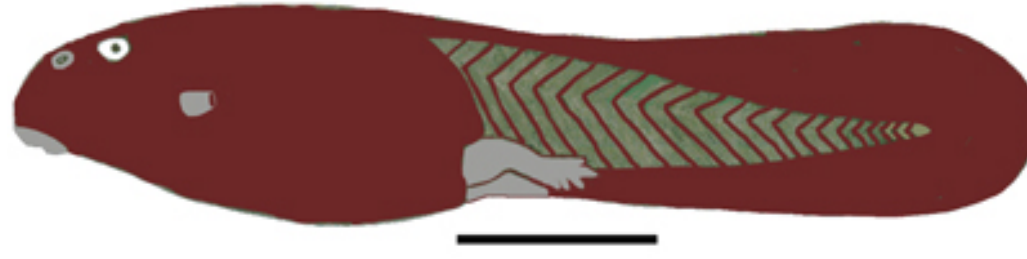

(a)

(b)

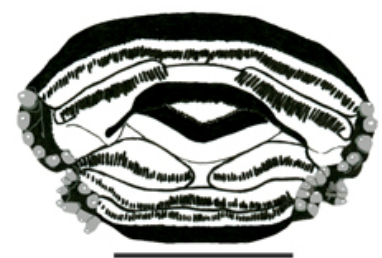

Figura 2. a) Vista lateral de la larva de Bufo cophotis en el estadio 36 . Barra $=5 \mathrm{~mm}$. b) Vista frontal del disco oral de larva de Bufo cophotis en el estadio 36 . Barra $=1 \mathrm{~mm}$. 
Hábitat: no se tienen datos del hábitat de los especimenes procedentes de Ancash, pero Duellman reporta, para larvas de esta especie, una coloración negra en vivo y, como hábitat, un riachuelo cubierto de hierbas ubicado en un páramo a $3500 \mathrm{~m}$, al sur del Abra Quilish, provincia Cajamarca, departamento Cajamarca (notas de campo de William E. Duellman, 1990).

\section{Discusión}

Los caracteres de la morfología externa larval dan soporte a la hipótesis que $B$. arequipensis no debería tener estado específico sino ser considerado como un sinónimo de B. spinulosus (Córdova, 1999). La larva de $B$. arequipensis es indistinguible en los caracteres externos discretos y se traslapa en todas las proporciones morfométricas examinadas, de las larvas de los taxones nominales B. flavolineatus y B. trifolium (Tabla 3). Además, Córdova (1999) encuentra en cromosomas que la coloración convencional, patrón de bandas $\mathrm{C}$, y localización de las regiones organizadoras del nucleolo de los taxones $B$. arequipensis, $B$. flavolineatus, $B$. spinulosus y $B$. trifolium muestran el mismo cariomorfo-cariotipo. Si $B$. flavolineatus y $B$. trifolium son considerados sinónimos junior de $B$. spinulosus, entonces la evidencia de la morfología externa larval y cromosómica apoyan la hipótesis que $B$. arequipensis también es un sinónimo de B. spinulosus.

La larva de $B$. limensis se diferencia de la larva de $B$. spinulosus por presentar un espacio mucho más angosto en la segunda fila de dentículos del labio anterior (Tabla 3) (fig. $1 \mathrm{~b}$ en Angulo y Aguilar, 2003), y por la fórmula de filas de dentículos labiales (2(2)/3[1]). Este carácter es independiente del estadio larval de los especimenes examinados (Tabla 1). Por otro lado, algunos individuos de $B$. limensis son de mayor longitud total que las otras larvas del grupo spinulosus, pero este carácter es variable con respecto al hábitat. En estadios similares, las larvas de $B$. limensis que habitan pozas temporales son de menor tamaño que las de pozas permanentes (observ. pers. del primer autor). Además, los renacuajos de $B$. limensis de pozas temporales tienen una longitud similar a las larvas de B. spinulosus en estadios comparables (observ. pers. del primer autor). Por eso, la longitud total larval no correspondería a un carácter útil para distinguir a $B$. limensis de las otras larvas de este grupo.

La larva de $B$. cophotis se diferencia de la de $B$. limensis y $B$. spinulosus por la coloración marrón oscura que es uniforme en el cuerpo y cola, presentar la parte más alta de la cola en su último tercio, y por la fórmula de filas de dentículos labiales (2(2)/3(1)). Por otro lado, la larva de $B$. corynetes y $B$. vellardi Leviton y Duellman siguen siendo desconocidas.

\section{Clave para las larvas conocidas de las es- pecies peruanas del grupo Bufo spinulosus}

1a. Color (en líquido preservativo) del cuerpo y cola pardo oscuro; altura máxima de la cola ubicado en su último tercio; LTRF igual a 2(2)/3(1)

\section{B. cophotis}

1b. Color (en líquido preservativo) del cuerpo diferente de la cola; altura máxima de la cola ubicado aproximadamente en la mitad de su longitud; LTRF diferente a $2(2) / 3(1)$

2

2a. Ancho del espacio en la segunda fila anterior de dentículos labiales es del 3 al 9\% del ancho de la segunda fila anterior de dentículos labiales; LTRF 2(2)/3[1]

\section{B. limensis}

2b. Ancho del espacio en la segunda fila anterior de dentículos labiales es del 17 al 29\% del ancho de la segunda fila anterior de dentículos labiales; LTRF 2(2)/3

$B$. arequipensis, B. flavolineatus y B. trifolium.

\section{Agradecimientos}

El primer autor agradece de manera especial al Ing. Alejandro Aguilar por su constante e invalorable apoyo. A Claudia Reis, Marco Méndez, Edgar Lehr y Jesús H. Córdova por la revisión de este manuscrito. A Jesús $H$. Córdova, Curador del Departamento de Herpetología del Museo de Historia Natural de la Universidad Nacional Mayor de San Marcos, que muy gentilmente brindó espacio 
y acceso a los especímenes. A Paúl Velazco por su apoyo en la obtención de la literatura. A Mikael Lundberg y Elías Ponce por su colaboración en el trabajo de campo.

\section{Literatura citada}

Altig, R. y McDiarmid, R.W. (1999): Body plan. Development and Morphology. En: McDiarmid, R.W. y R. Altig (Eds): «Tadpoles. The Biology of Anuran Larvae». Chicago, the University of Chicago Press. 444 pp.

Angulo, A. \& C. Aguilar 2003. The Tadpole of Bufo limensis (Werner, 1901). Amphibia-Reptilia, (24): 400-405.

Cei, J. M. 1972. Bufo of South America. En: Blair, W. F. (Ed): «Evolution in the genus Bufo». University of Texas Press. $459 \mathrm{pp}$.

Cei, J.M. 1980. Amphibians of Argentina. Monitore zool. ital., (2): 165-168.

Córdova, J.H. 1999. On karyomorphs, cladistics and taxonomic status of the Bufo spinulosus species group (Amphibia : Anura) in Peru. Stuttgarter Beitr. Naturk. Ser. A., (600): 1-28.

Duellman, W. E. y R. Schulte. 1992. Description of a new species of Bufo from northern Peru with comments on phenetic groups of South American toads (Anura: Bufonidae). Copeia, (1): 162-172.
Frost, D.R. 2002. Amphibian Species of the World: An online reference. V. 2.21 (15 July 2002). Electronic database available at

http://research.amnh.org/herpetology/amphibia/ index.html.

Gosner, K.L. 1960. A simplified table for staging anuran embryos and larvae with notes on identification. Herpetologica, (16): 183-190.

Haas, W. 2002. Beitrag zum taxonomischen Status von Bufo trifolium Tschudi, 1845 und Bufo spinulosus flavolineatus Vellard, 1959 sowie zur Biologie von Bufo spinulosus Wiegmann, 1834. Salamandra, (3): 155-164.

Lehr, E. 2002. Amphibien und Reptilien in Peru. Natur und Tier Verlag, Münster. 208 pp.

Martin, R. F. 1972. Evidence from osteology. En: Blair, W. F. (Ed). «Evolution in the genus Bufo». University of Texas Press. 459 pp.

Maxson, L. R. 1984. Molecular probes of phylogeny and biogeography in toads of the widespread genus Bufo. Mol. Biol. Evol., (4): 345-356.

Sinsch, U. 1986. Anfibios de la sierra central del Perú. Una clave de identificación para adultos y larvas. Boletín de Lima, (45): 23-32.

Sinsch, U. 1990. Froschlurche (Anura) der zentralperuanischen Anden: Artdiagnose, Taxonomie, Habitate, Verhaltensökologie. Salamandra, (26): 177-214.

Vellard, J. 1959. Estudios sobre batracios Andinos V-El género $\underline{B u f o}$. Mem. Museo Hist. Nat. J. Prado, (8): 1-48 